\title{
Procesos que obstaculizan la inclusión en la educación secundaria obligatoria. Muchas sombras y todavía pocas luces
}

\author{
Dolors Forteza-Forteza y Francisca Moreno-Tallón
}

\begin{abstract}
RESUMEN
Las medidas extraordinarias de atención a la diversidad en la Educación Secundaria Obligatoria (ESO) son objeto de la investigación que presentamos en este artículo. Con diferente denominación según la comunidad autónoma, nos centramos en una de estas medidas, el Programa de Intervención Educativa (PIE), cuyos destinatarios son alumnos en situación de riesgo personal o social. Desde una perspectiva metodológica cualitativa analizamos un caso para profundizar en las vivencias de alumnos con dificultades de comportamiento que forman parte de un PIE. Se recogió la información a través de entrevistas y la observación, y el análisis de documentos fue una técnica complementaria para garantizar la triangulación de fuentes de registro.

Los resultados ponen de manifiesto el complejo contexto de tensiones que atraviesan los alumnos con dificultades de comportamiento en un programa específico. Las conclusiones interpelan al diálogo con el alumnado, a intervenciones educativas en el aula, a la aceptación de las biografías personales y al derecho de que se satisfagan sus necesidades junto con los iguales, para eliminar las barreras y obstáculos que estos alumnos viven diariamente durante su trayectoria escolar. Se requieren respuestas educativas significativamente contextualizadas para favorecer la inclusión de todo el alumnado.
\end{abstract}

Palabras Clave: educación secundaria, exclusión, dificultades de comportamiento, medidas extraordinarias, educación inclusiva.

\section{Processes that hinder inclusion in Compulsory Secondary Education. Many shadows and still few lights}

\section{ABSTRACT}

The extraordinary measures of attention to diversity in Compulsory Secondary Education (ESO) are the subject of the research presented in this article. With a different denomination according to the autonomous community, we focus on one of these measures, the Educational Intervention Program (PIE), whose beneficiaries are students at personal or social risk. From a qualitative methodological perspective we analyze a case to deepen the experiences of students with behavioral difficulties that are part of a PIE. Information was collected through interviews and observation, and document analysis was a complementary technique to ensure the triangulation of registry sources.

The results highlight the complex context of tensions that students with behavioral difficulties go through in a specific program. The conclusions call for dialogue with students, for educational interventions in the classroom, for the acceptance of personal biographies and the right to meet their needs with peers, to eliminate the barriers that these students live daily during their school career. Significant contextualized educational responses are required to encourage the inclusion of all students.

Keywords: secondary education, exclusion, behavioral difficulties, extraordinary measures, inclusive education.

\section{Introducción}

En este estudio nos centramos en adolescentes con dificultades de comportamiento (DC) en la ESO. Una etapa en la que los conflictos y problemas se incrementan, al tiempo que proliferan diferentes respuestas educativas para atender, supuestamente, las necesidades de estos alumnos. Profundizar en cómo se sienten cuando son ubicados en propuestas específicas de intervención y analizar las barreras que impiden u obstaculizan su participación y progreso, es la finalidad que perseguimos.

Hacemos referencia a dificultades de comportamiento cuando se dan rasgos tales como intensidad emocional elevada, impulsividad, persistencia negativa, resistencia inicial, hiperactividad, entre otros (Saumell, Alsina y Arroyo, 2011). Los estudiantes con DC, de acuerdo con Leeuw, Boer, Bijstra y Minnaert (2017), presentan dificultades en la regulación 
efectiva de sus interacciones sociales y un comportamiento y/o funcionamiento emocional que puede interferir en su desarrollo y en la vida de los demás. Dificultades que pueden influir de manera negativa en las oportunidades de participación social positiva, tal y como señala Avramidis (2010).

No obstante, la conducta de los estudiantes puede variar no solo en función de algunas características personales, sino también de factores contextuales que emergen en cualquier momento de su escolaridad (Sandoval y Simón, 2007).

Considerar estos factores es esencial en el marco de la educación inclusiva porque, como nos recuerdan Danforth y Smith (2005), los comportamientos disruptivos que se dan en el aula tienen que hacer cambiar las bases sobre las que se sustentan los centros escolares. Asimismo, deben modificarse las actitudes puesto que la frustración inicial que experimentan estos alumnos a menudo se debe al hecho de que son considerados "malos" y "perturbadores".

Por consiguiente, los antecedentes, condiciones y factores del contexto, además de originar necesidades (González, 2005), pueden situar a los alumnos en zonas de vulnerabilidad tanto social como educativa, si el profesorado y la propia cultura de los centros se fija meramente en sus fracasos y no en cómo satisfacer sus necesidades. Dicho en otras palabras, según el contexto, los alumnos con dificultades de comportamiento, es probable que estén en riesgo de exclusión en el transcurso de la educación obligatoria.

Considerando que "la exclusión educativa y el fracaso escolar no son fenómenos naturales" (Rodríguez, Álvarez y Moreno, 2009, p.176), son ya muchos los estudios que han puesto de manifiesto que tras el fracaso de los estudiantes en la ESO se velan itinerarios escolares cargados de problemáticas que se inician en la educación primaria, y en la secundaria obligatoria se exteriorizan con un progresivo desapego hacia los estudios. Desapego que, en el caso que nos ocupa, es consecuencia, en gran parte, de la estructura rígida que caracteriza esta etapa educativa en múltiples dimensiones (curriculares, de formación del profesorado, organizativas, etc.), y tiene igualmente "implicaciones políticas, puesto que permite interrogar sobre los procesos institucionales que contribuyen al desarrollo de una trayectoria de desafección escolar [...]" (García, Casal, Merino y Sánchez, 2013, p.71).

En esta misma línea se manifiestan, con anterioridad, Mena, Fernández-Enguita y Riviére (2010), cuando hablan de la desvinculación gradual o del desenganche que se produce por parte del alumnado con respecto a la institución.

En nuestro país, frente a las elevadas tasas de fracaso escolar (Roca, 2010; Aramendi, Vega y Santiago, 2011), las administraciones educativas desarrollan una variedad de programas específicos para dar respuesta a la diversidad del alumnado que tienen un componente de exclusión, sin cuestionarse los procesos desencadenantes de la segregación educativa de aquel alumnado que por alguna circunstancia se considera diverso, distinto o especial (Moliner, Sales, Ferrández, Moliner y Roig, 2012).

Fulcher (1989), en su momento, ya consideró la posibilidad de que se produjeran nuevas formas de segregación en los entornos normalizados, mediante prácticas que dividen. Prácticas que se pueden convertir en opciones remediales que generan vías de escolarización paralelas para un sector del alumnado, provocando en algunos casos lo que Young (2000) designa "exclusión interna", originándose "zonas de discriminación".

Para Escudero, González y Martínez (2009), se trata de alumnos a los que no se les deja del todo fuera, pero tampoco se les incluye de modo efectivo en un currículo y enseñanza de calidad que les ayude a lograr los aprendizajes necesarios.
Las medidas que se planean para estos alumnos, y específicamente para los que presentan dificultades de comportamiento, si bien tienen como fin explícito que obtengan el título de graduado en educación secundaria, ultiman la etapa de la ESO sin éxito. Y aunque estas medidas o propuestas pueden considerarse para algunos alumnos como "segundas oportunidades" -en palabras de Escudero y Martínez (2011)lo cierto es que muchos otros se ven abocados al fracaso y al abandono de su formación básica.

\section{Método}

El trabajo que presentamos se enfoca desde un método cualitativo de investigación, el estudio de caso, para dar respuesta a dos objetivos: (1) Conocer la experiencia educativa de los alumnos con dificultades de comportamiento en un grupo específico; (2) Indagar en las percepciones y expectativas del profesorado respecto a estos alumnos en distintas situaciones de aula.

Desde este marco tres son los instrumentos que se han contemplado para recabar información: la entrevista, la observación no participante y análisis de documentos. Mientras que la entrevista y la observación son las fuentes principales de registro, los documentos ofrecen informaciones del contexto y de contraste, lo que permite la triangulación.

Se realizaron entrevistas a los alumnos y al profesorado, a los que se facilitó la transcripción, y se informó al director de los resultados después de un primer análisis de la información recabada.

Se observaron las clases de Ciencias Naturales, Lengua y Literatura Castellana y Educación Física. Decisión que estuvo condicionada por cuestiones de compatibilidad horaria. Se hizo uso de un formato abierto de notas de campo, recogiendo información tanto de los alumnos como del profesorado.

Por otra parte, se analizó el Plan de Convivencia del centro y las programaciones. Estas pruebas documentales se convirtieron en un apoyo útil para la observación y para el análisis de la información recogida.

\section{El centro y los participantes}

El instituto se sitúa en una zona turística de Mallorca, y cuenta con 1100 alumnos y 117 profesores. El aula del grupo específico. no se ubica físicamente donde se encuentran todas las aulas sino en un anexo entre el patio y las instalaciones deportivas.

La muestra está formada por 5 profesores y 12 alumnos. De estos, 4 se dieron de baja después de expulsiones reiteradas de larga duración y uno, al cabo de tres meses, se incorporó a un grupo de $2^{\underline{o}}$ de la ESO a petición de la familia. Finalmente, el programa de intervención educativa (PIE) se configuró con siete alumnos que repetían $2^{\circ}$ de ESO, y que a lo largo del curso escolar tenían que cumplir los 16 años, más uno que era repetidor de $1^{\mathrm{O}}$ de ESO.

\section{Perfil del profesorado y alumnado}

La profesora de Lengua y Literatura Castellana (PLLC) es licenciada en Filología inglesa con 10 años de experiencia docente. Es su primer año en el centro.

El profesor de Ciencias Naturales (PCN) es a la vez uno de los orientadores del instituto. El hecho de que impartiera las clases de esta materia era una medida adoptada por la dirección, ya que el mayor número de horas lectivas de los alumnos de este programa tenían que ser cubiertas por el profesorado del 
Departamento de Orientación. Es licenciado en Psicología y tiene 7 años de experiencia docente. Es su segundo año de trabajo en este programa y el primero que está en este centro.

Los dos profesores de Educación Física son licenciados en Ciencias de la Actividad Física y del Deporte, ambos también de nueva incorporación. El profesor de Educación Física 1 (PEF1) es oficialmente el profesor de los alumnos del PIE; tiene dos años de experiencia como docente y es la primera vez que participa en este tipo de programas. La profesora de Educación Física 2 (PEF2) es la titular de un grupo-clase de $2^{\circ}$ de ESO; tiene 4 años de experiencia docente, de los cuales 2 en el programa. Debido a la falta de motivación de los alumnos del PIE hacia la asignatura, propuso que trabajaran conjuntamente con todos los alumnos.

Respecto a los alumnos destacar que únicamente hay una adolescente, en adelante identificada como alumno 1.

A1: de nacionalidad inglesa con 16 años recién cumplidos. En el instituto formó parte del Programa de Acogida Lingüística y Cultural (PALIC). Repitió $1^{\circ}$ de ESO y es remarcable que vuelve a realizar por segunda vez el programa PIE.

A2: no hay datos destacables de su Educación Primaria, pero en el instituto repite $1^{\circ}$ y $2^{\circ}$; cuando debe realizar por segunda vez el curso de segundo pasa a formar parte del PIE.

A3: es de origen inglés. Repitió $6^{\circ}$ curso. Al empezar la secundaria repite $1^{\underline{o}} \mathrm{y}$, posteriormente, ya se incorporó al programa PIE.

A4: inglés de nacimiento realizó toda su escolarización primaria en España. Ha repetido $6^{\circ}$ de Primaria y $1^{\circ}$ de la ESO; pasa al PIE después de haber cursado segundo.

A5: durante su escolaridad repitió $2^{\circ}$ de primaria y de ESO. Su madre falleció hace unos años y es la hermana un poco mayor que él (20 años) la que se encarga de los menores.

A6: es un alumno diagnosticado con TDAH. En primaria repitió $2^{\underline{o}}$ y en el instituto es su segundo año en $1^{\circ}$ de ESO; no obstante ha pasado a formar parte del programa PIE, aun siendo un alumno más joven que el resto.

A7: repitió $3^{\circ}$ en primaria y cuando se incorporó al instituto repitió $1^{\circ}$.

A8: es un alumno que tiene problemas con sustancias estupefacientes. Dejó el programa durante el tercer trimestre por una larga expulsión.

\section{Resultados}

Nos encontramos ante un grupo de alumnos cuya exclusión se ha ido fraguando progresivamente. Ahora, bajo el paraguas de las medidas extraordinarias para ayudarles a adquirir los conocimientos básicos de la ESO -con el fin de obtener el título de graduado en educación secundaria, se encuentran en el margen; esa línea casi invisible que les separa cada vez más de los otros sus iguales y de vivencias normalizadas de aprendizaje.

Ya no solo están aislados (grupo específico), son también las propuestas que realiza el profesorado y la interacción que se produce entre este y los alumnos que no logran ningún beneficio para el aprendizaje.

El ambiente de clase está deteriorado. Un deterioro que se justifica por las actitudes y comportamientos de los alumnos: hablar durante las explicaciones, negarse a realizar las tareas de lápiz y papel, llegar tarde, agresiones a compañeros, insultos, comer en clase, levantarse de la silla, balancearse, faltar al respecto, etc., que son la causa del 'no aprendizaje', como si la enseñanza (el profesorado) no tuviera nada que ver en este proceso.

El 'mal' comportamiento es vivido por los profesores con resignación ('nos ha tocado este grupo'); la etiqueta de "malos alumnos" es persistente, casi definitiva para algunos, y tiene el efecto de profecía que se autocumple: el profesorado asiste a la clase con el prejuicio de que tendrán un comportamiento muy desfavorable (ya están marcados) y el alumnado responde con las conductas esperadas. Se crea, por consiguiente, un ambiente poco propicio para enseñar y para aprender, algo que sucede cotidianamente en el aula.

Una cotidianeidad que de partida ya es excluyente, no solo por formar parte de una medida excepcional sino también por las implicaciones que tiene en el proceso de escolarización de estos alumnos. No participan en las actividades lúdicas o extraescolares que realizan sus iguales; no participan, entre otros aspectos, porque la ubicación de su aula se encuentra totalmente separada y diferenciada de las demás aulas, aislada. Los alumnos son conscientes de por qué están en ese espacio y son contundentes sus manifestaciones:

La clase está separada del instituto y nunca hacemos nada que hacen los demás segundos de ESO. Me gusta más un $2^{\circ}$ de ESO normal porque estás con más gente; es que yo soy la única chica en este grupo (A1).

La clase es lo peor [...]. Sólo hay eco, tú dices una palabra y hay eco. La música del polideportivo que se oye todo el rato. Es imposible hacer clase, luego quieren que te concentres y trabajes, no puedes... (A4).

La clase [...] es como una jaula, hay eco y cuando hacen Educación Física nos miran todo el rato como si fuésemos anormales (A5).

Lo más negativo de este año ha sido pasar todo el curso ahí en esa clase (A7).

Una de las profesoras expresa sobre esto:

Lo peor que me ha parecido es que estén allí apartados completamente a nivel espacial, totalmente apartados y que las condiciones de la clase sean bastante terribles en cuanto a condiciones sonoras, frío y calor [...], tampoco les han dejado hacer salidas, no les han dejado participar en cosas que otros sí han hecho. [...] Totalmente apartados de los compañeros porque... ¿oye? también se tienen que relacionar, ¿no? (PLLC).

Los alumnos, por su parte, denuncian su "no presencia y participación" en las actividades del centro; se prescinde de ellos marcando la diferencia entre el alumnado, en general, y el que está en los márgenes, fuera:

Yo he visto como todos los de segundo se van de excursión menos nosotros, no nos dejan ir de excursión ni nada y no sé por qué. No hacemos lo de ecología que lo hace todo el instituto (A1).

No hemos hecho ninguna excursión, apartados de todos los grupos de segundo. Hicieron pruebas de Educación Física en el patio todos los segundos y nosotros ninguna, la charla de las drogas que vino súper tarde que casi se olvida de nosotros. $\mathrm{Y}$ yo con esto me siento mal y me enfado con la tutora... (A2).

¡Ah! Y no salimos de excursión pero me parece que eso es normal, sí porque la liaríamos, supongo. Es que no confían en nosotros, si nos dieran una oportunidad pues acordamos entre nosotros que nos tenemos que portar bien y saldría todo bien, no nos han dado la oportunidad y la hemos pedido mil veces (A4).

[...] nosotros dijimos "te prometemos que no haremos nada" y nos dice "no, no, no confío en vosotros". Es injusto 
porque los demás se pueden ir de excursión y nosotros no y luego nos dice que no somos especiales, que somos normales como los demás, pues si somos normales nos dejaría ir de excursión (A5).

Este proceso de segregación interna tiene un gran impacto en la autopercepción y en lo que piensan respecto a cómo les perciben los demás. Lo que expresan estos tres alumnos es una evidencia de esta afirmación:

Siempre piensan mal de nosotros: no sabes estudiar, nunca haces nada, no vas a hacer nada en tu vida (A1).

Y los demás del instituto nos ven como tontos, las cosas como son. Tontos y que vamos a acabar mal debajo de un puente o algo así. Y los profesores también nos ven mal. Yo es lo que pienso (A2).

Te tratan como a un tonto la gente, profes y alumnos. Te tratan diferente... hay profes que te tratan con cuidado porque a lo mejor piensan que luego te enfadas y la lías... (A4).

La percepción de que son "tontos" y que les perciben como tales, es reafirmado por este último alumno al referirse a los libros de texto:

También nos toman como tontos porque los libros son nuevos, son de estos que se puede escribir en ellos y eso para mí es de retrasado. Tenemos los libros diferentes, te lo explican más fácil, mucho más de tontos (A4).

Se les percibe y se les trata como 'alumnos problema' (conflictivos), motivo más que suficiente para ubicarles en otro lugar, un programa específico. Para el profesorado esta medida extraordinaria es la mejor opción para no perjudicar al resto de alumnos del aula regular:

Tienen una actitud muy negativa. Ellos se quejan de que realmente están todos juntos y que son los apartados pero estoy segura de que en un grupo normal hubieran tenido la misma actitud y les hubieran seguido dos o tres y hubieran hecho lo mismo prácticamente (PLLC).

Los profesores que imparten las diferentes asignaturas en este grupo, además de no coordinarse (así lo manifiestan), no se plantean otro tipo de intervención ni en el aula ni en el centro para movilizar su aprendizaje y mejorar su rendimiento, tampoco su interacción con el resto de alumnos. Aunque sí ven en esta medida 'daños colaterales': "Yo creo, que al hacer así como un gueto, la actitud empeora. Es como retroactivo, o sea uno que se comporta medianamente bien se une al grupito de los que se portan mal y acaba portándose mal" (PEF1). Lo que preocupa: el comportamiento.

Las evidencias muestran que los alumnos no son aceptados, han vivido y viven una trayectoria de desencuentros, algo que va calando en ese sentimiento de "no pertenencia". Un alumno resume así la situación: "Nosotros somos una cosa aparte. Una clase aparte, como no sé... de marginados, yo no me he sentido bien" (A7).

Evidencias que del mismo modo ponen al descubierto el trato diferente que reciben: no tienen oportunidades, se les ignora, se desconfía de ellos, entre otros muchos aspectos, y su bienestar se ve afectado, así como su autoestima.

No obstante, es importante hacer referencia a la reacción positiva que estos alumnos tienen cuando se les hacen propuestas en las que tienen oportunidades de participar con los iguales. Es el caso de las sesiones de Educación Física, en la que el grupo comparte actividades con una de las clases de $2^{\mathrm{o}}$ de ESO. Mostraron más interés y motivación, en palabras de la profesora:

La actitud del alumnado cambia mucho. Su clase era súper apática, no querían hacer nada, cuando se juntaron se han sentido más motivados. [...] Hay aspectos a mejorar, todo lo que hace referencia a hábitos les cuesta mucho, pero después también te sorprenden porque te vienen a decir "me lo he pasado muy bien con el baile" y al principio decían "yo paso de bobadas". Ahora te dicen que les ha encantado bailar rock. Son chicos que tienen carencias pero se puede mejorar, juntos pueden mejorar (PEF2).

El proceso de experimentar y compartir vivencias no estuvo exento de problemas aunque mereció la pena según manifiesta la misma profesora. Alude a un alumno muy conflictivo al hacer la siguiente reflexión: “Cuando juntamos el grupo con el otro, A6 empezó a colaborar. Uno malo lo podemos cambiar junto con los demás pero ¿si todos son malos?". Y está satisfecha del trabajo conjunto con el otro profesor de su área: "Yo he estado muy bien trabajando con él porque he visto otros ejercicios, los he copiado $\mathrm{y}$ los he hecho en mis clases porque pensaba si sale con estos alumnos imagina con los otros" (PEF2).

Las historias escolares de los alumnos revelan que sus dificultades de aprendizaje empiezan a visibilizarse en la educación primaria culminando con la repetición de algún curso. Recuerdan situaciones de su paso por esa etapa como estas que siguen a continuación:

Siempre estaba en mi mundo, bueno desde quinto o eso. Es que siempre me ha costado concentrarme. Me hacían sentar delante y salía de la clase en algunas asignaturas (A4).

Cuando me aburría era porque si comienzan a explicar y no lo entiendo pues los profesores no me lo querían explicar, porque se lo preguntabas un montón de veces, entonces pues ya paso de preguntar y cuando no entendía una cosa me quedaba dibujando o durmiendo. No me lo explicaban porque me costaba mucho entenderlo (A5).

Una de las características más comunes entre estos alumnos en el transcurso de la educación secundaria es la falta de motivación. El contexto no les ayuda y mucho menos las metodologías del profesorado que se dirigen, sobre todo, a un alumno "estándar" inexistente. Llegan a la ESO con dificultades de aprendizaje y no conectan con la estructura de clase lineal: explicación, copiar de la pizarra, ejercicios del libro y deberes en casa. Afloran los comportamientos disruptivos a los que les sigue una respuesta sancionadora (expulsión) que les aleja de la actividad cotidiana del aula y cuando retornan no se 'enganchan'; empieza de nuevo el círculo de la desmotivación. ¿No es acaso esta cadena un proceso de exclusión? Las palabras de este alumno refiriéndose a los profesores de la ESO lo reflejan de alguna forma:

No ha habido ninguno que fuera como alguno del cole de bien, de buenos. $\mathrm{Y}$ hay peores. Han sido más bordes, como que pasan, nada más que dan la clase a 5 o 6 y de los demás pasan. Claro, pasaban de mí como que yo no quería hacer nada porque no sé, algo sabía hacer pero en la mayoría tenía dudas (A7). 


\section{Discusión y conclusiones}

Lo que se ha descrito hasta aquí muestra un escenario desfavorable para los alumnos que han participado en el estudio. El sentimiento de rechazo es unánime, son historias de exclusión y de ausencia de reconocimiento. Se trata de alumnos que habitualmente se sienten desprestigiados a causa de las etiquetas que se les atribuye; uno de ellos es contundente cuando expresa: "la profesora de castellano nos dice ¡tú no sirves para nada! Y yo no sé por qué lo dice [...] aunque creo que es verdad y es la única que nos dice la verdad". Historias que se caracterizan por la falta de confianza del profesorado en sus capacidades, tanto en el pasado como en el momento en el que transcurre el trabajo de campo. Los alumnos no quieren estar ahí, en un grupo aparte, y los docentes tampoco, simplemente 'les ha tocado'.

Estamos frente a alumnos estigmatizados; se les ha asignado una serie de características que les ha llevado a formar parte de un programa extraordinario, legitimando así el trato excluyente que han recibido con efectos indeseados para su aprendizaje, rendimiento y su trayectoria vital. Teniendo en cuenta que algunos estudios afirman que existe una correlación directa entre las dificultades de comportamiento y el hecho de que estos alumnos se sientan incapaces de afrontar los rigores académicos que se exigen en la educación secundaria (Garner, 2005; Garner y Davies, 2007).

Para enseñar hace falta sumergirse en la "cultura de la confianza, dirá López Melero (2004, p.21), que hay que ir construyendo teniendo en el epicentro el valor de la diferencia, lo que requiere profundas transformaciones de los centros para hacer posible una educación para todos. Una educación que debe proporcionar, independientemente de cualquier diferencia personal, social o emocional, los recursos que necesiten los niños y adolescentes (Florian, Young y Rouse, 2010) sin tener que ofrecer propuestas educativas paralelas a las ordinarias. En este sentido, lo trascendente es focalizar los análisis y los cambios en los microsistemas (los centros educativos) y no hacia las personas.

Como indica Vilaró (2007), la clave está en promover el diálogo, en convencer al adolescente que hable con el adulto y convencer al adulto que escuche al alumno, que se expliquen, que se conozcan un poco más. Es decir, establecer una relación positiva entre el alumnado y el profesorado que se sustente en el respeto y la comunicación (Meyers, 2009).

El estudio llevado a cabo por Álvarez, Álvarez, Castro, Castro y Fueyo (2008), sobre las actitudes del profesorado de educación secundaria hacia la inclusión, hace hincapié en que las opiniones de los docentes variaban en función del colectivo de alumnos de que se tratara. $\mathrm{Y}$ pone de relieve que los que presentan dificultades de comportamiento son los menos aceptados. En este sentido, habría que ahondar en la falta de diálogo que se produce entre profesores y alumnos y la falta de reconocimiento de los primeros respecto a los segundos. Porque tal y como expone Vilaró (2007):

Detrás de muchos incidentes, insultos y agresiones a adultos seguro que hay una historia previa de malos entendidos, menosprecios, mala educación, más malos entendidos, todavía más menosprecios (...) que no tengan "modos" no quiere decir que no tengan nunca razón. Muchos adolescentes que plantan cara a los profesores lo hacen desde la insignificancia que se les ha hecho sentir (p.198).

Para promover el aprendizaje el alumnado debe sentir que vale la pena aprender. $Y$ esto converge con el sentimiento de valía y las relaciones de pertenencia, amistad y participación en el grupo-clase y en el centro (Echeita et al., 2014). Para los mismos autores:

Lo contrario, esto es, todo aquello que, como resultado dela forma de organizar la enseñanza y el aprendizaje, contribuya al desarrollo, en determinados alumnos, de sentimientos y situaciones de fracaso reiterado, aislamiento, marginación, minusvalía o de exclusión deben ser considerados como barreras de primer orden para el aprendizaje (p.32).

Otra cuestión a subrayar es el paso de una etapa educativa a otra. De acuerdo con Martínez (2011, p.168) "la transición de la etapa primaria a la secundaria es cuando el alumnado más vulnerable corre los mayores riesgos de ser excluido del sistema educativo ordinario". Efectivamente, en nuestro estudio lo hemos constatado; los alumnos con dificultades de comportamiento, que ya arrastran un historial de fracasos (han repetido en las dos etapas) son dirigidos, casi inevitablemente, hacia alternativas educativas no regulares, y el PIE es una de ellas cuando iniciamos el estudio. Aquí entran en juego las actitudes del profesorado y sus expectativas:

El profesor cree que ya no se puede hacer nada y el alumno piensa que no merece la pena cambiar. Esta espiral de expectativas negativas contribuye a reforzar el comportamiento inadaptado. A veces, tampoco el profesor sabe cómo resolver el problema por un método que no sea la sanción. Hace falta conversar y pactar para intentar modificar esta dinámica (Marchesi, 2004, p.149).

Al hilo de las bajas expectativas: dañan cualquier proceso de interacción. Las evidencias así lo han puesto de manifiesto; los alumnos perciben que no son valorados por sus profesores, a lo que responden con conductas que no saben cómo gestionar, produciéndose un deterioro progresivo de la relación. El círculo se cierra en sí mismo. Recordemos las palabras de uno de los alumnos ya mencionadas con anterioridad: "Siempre piensan mal de nosotros: no sabes estudiar, nunca haces nada, no vas a hacer nada en tu vida" (A1).

En esta línea, otras investigaciones han mostrado que estos alumnos perciben la relación con sus profesores como el factor que más influye a la hora de establecer un comportamiento de acuerdo con la norma en el aula (Turner, 2000). Pese a las evidencias se mira hacia otro lado, los alumnos son los portadores de dificultades, de defectos, lo que justifica la segregación en un grupo especial; un itinerario que les conducirá definitivamente a destinos muy desiguales, utilizando palabras de Slee (2012).

Queremos poner énfasis en las identidades de los alumnos, en sus biografías personales. Como ha quedado reflejado, la educación secundaria para un sector del alumnado fracasa (y si falla para los que tienen ciertas dificultades, falla también para todos), y lo hace porque no reconoce las necesidades de los estudiantes, no se les brinda oportunidades para aprender que despierten su interés y motivación (Birbili, 2005). Por el contrario, lo que prima son las bajas expectativas que el profesorado tiene de estos alumnos ("los repetidores", "los malos", "disruptivos", ...), a los que, sucesivamente, se les van poniendo barreras a su participación y progreso, acabando la escolaridad obligatoria en un grupo específico sin conseguir graduarse.

Al hilo de las barreras, consideramos que el fracaso de los alumnos no puede entenderse en términos individuales (lo que no hacen, lo que no saben, su conducta...), son necesarias explicaciones globales, sistémicas: 
[...] el fracaso escolar puede leerse en relación con las dificultades de las instituciones educativas por encontrar o buscar soporte en la comunidad; en relación con la propia configuración de los estudios en la educación secundaria; en relación con el desconocimiento de las historias personales y con no tenerlas en cuenta en los requerimientos y dinámicas escolarizadas; en relación con la distancia familia-institución; en relación con componentes interculturales, religiosos; en relación también con el origen social que determina las posibilidades y la igualdad de oportunidades; en relación con las fracturas personales, y con la construcción de identidades complejas; en relación con la distancia hacia las culturas infantiles y juveniles presentes en el escenario social; en relación con el género, etc. (Hernández y Tort, 2009, p.6).

De ahí la importancia de analizar las trayectorias escolares; con ello queremos incidir en la repetición de curso pues es un hecho relevante en tanto que la mayoría de los alumnos participantes han repetido ya en primaria $y$, posteriormente, en secundaria. Esto confirma que el fracaso se está forjando en edades tempranas, no es "un fenómeno puntual o un desenlace final" dirán Mena et al. (2010, p.121), sino "un proceso lento que acompaña al alumno a lo largo de su vida escolar o de una parte importante de ella" (p.121). Rué et al. (2006) ratifican esta idea en su investigación al aseverar que la desvinculación se intensifica en la educación secundaria pero comienza en la etapa que le precede. La cultura docente, en conjunto, no es capaz de compensar las carencias escolares de origen, como tampoco las de orden sociocultural; y remarcan que los factores organizativos de centro, las expectativas y las representaciones que tiene el profesorado con respecto a sus alumnos, el clima institucional, las modalidades de atención hacia el alumnado, entre otros, tienen un papel destacable en el grado de éxito escolar (Rué et al, 2006).

Respecto a las actitudes de los alumnos, calificadas a menudo de desafiantes, negativas y perturbadoras, en la investigación realizada por Fernández-Enguita, Mena y Riviére (2010), se constata que dichas actitudes (que denominan antiescolares), "salvo tal vez para grupos muy determinados y culturalmente muy distantes de la corriente principal, no tienen su origen en la familia, sino en la escuela, donde al descuido de la institución se une la dinámica autoalimentada del grupo de pares en la adolescencia; esta situación favorece la conversión de conductas episódicas en estrategias sistemáticas de rechazo" (p.186). Los mismos autores son concluyentes con la repetición de curso, aspecto este que caracteriza al grupo de alumnos que ha participado en el estudio; es, con toda probabilidad, un indicador de la falta de capacidad de la escuela para responder a la diversidad del alumnado: "la repetición puede considerarse un espléndido predictor y casi con seguridad una causa del abandono" (p.188).

Volviendo a la estigmatización que sienten los alumnos al estar en un grupo separado, el de los "tontos" (como indican ellos), nos lleva a reflexionar sobre lo que se esconde detrás de una medida de diversificación como es el PIE: la incompetencia institucional entendida en un sentido amplio (tanto de centro como de aula). También esta cuestión contrasta con la investigación de Fernández Enguita et al. (2010), al señalar las debilidades de estas medidas extraordinarias tales como:

el relativo estigma que acompaña a esos mismos cursos, (...) y la convicción mayoritaria entre los alumnos de que con esos grupos y medidas de diversificación se pierde más que se gana, sea por las expectativas institucionales o por las malas compañías. En general, el alumno no percibe en la diversificación una ayuda adicional ni mucho menos un esfuerzo especial de la escuela, sino una descalificación de su persona. Y aunque los profesores demandan más medidas de diversificación, todo indica que piensan en diferenciar los objetivos perseguidos con los alumnos, incluidos, por supuesto, los de la enseñanza obligatoria, no en diversificar los medios y los recursos para alcanzar unos mismos objetivos (p.199).

En síntesis, el fracaso que afecta a diferentes colectivos de alumnos, sobre todo en la ESO, es un problema complejo:

No solo porque priva a las personas del derecho a un bagaje de herramientas intelectuales, personales y sociales para poderse labrar un presente y futuro dignos, sino también porque representa una amenaza a las raíces mismas de la vida en común, la democracia auténtica, el progreso económico y social y, desde luego, la propia credibilidad y legitimidad del sistema educativo (Escudero y Martínez, 2012, p.177).

Y un problema complejo requiere una mirada sistémica, holística, para que el derecho a aprender sea para todos. Desde esta perspectiva, actuaciones que segregan al alumnado con dificultades no dejan de ser un mero "parche" que aumentan las barreras con las que estos alumnos se encuentran en su trayectoria vital, y escolar en particular.

El reto es la educación inclusiva. Una educación que pasa, como apunta Escudero (2012), por la mejora de los resultados escolares:

No nacen espontáneamente; han de ser social, institucional y personalmente construidos. Las mejoras y avances en los aprendizajes escolares no pueden ocurrir sin cambios profundos en el currículo escolar, en los procesos de enseñanza y aprendizaje, en la profesión docente, en el gobierno de los centros y en la administración y gestión del sistema (p.117).

O dicho de otra manera, progresar hacia la inclusión requiere, por una parte, voluntad política y acuerdo social basado en valores de equidad y justicia; $y$, por otra parte, formación del profesorado, cambios en el diseño y desarrollo del currículum, dotación y redistribución de los recursos humanos y materiales, además de decisiones valientes sobre la organización de los centros -en un marco caracterizado por la flexibilidad y la autonomía que promueva la participación de la comunidady sobre los procesos de enseñanza y aprendizaje, incidiendo especialmente en éste último (Durán y Giné, 2011).

La equidad, como afirman Domínguez, López y Vázquez (2016), sigue siendo uno de los eslabones más frágiles en el sistema educativo. La mudanza hacia planteamientos inclusivos choca con la arraigada visión reduccionista de que las medidas extraordinarias son las más beneficiosas para los alumnos con dificultades. Nutrirse de enfoques que abordan la diversidad homogeneizando las acciones educativas representa una incoherencia en los discursos de los propios centros, pero aún peor, si cabe, es suprimir la pluralidad individual dejando en los márgenes a aquellos alumnos que no responden al estándar.

Parafraseando a Pujolàs (2010), las prácticas que separan, aparentemente, en función de las necesidades de los alumnos con la intención de atenderles mejor, arrinconan la idea clave que se defiende en la educación inclusiva: "para perseguir 
una formación integral no solo para ellos, sino para todos, se requiere precisamente que tengan la oportunidad de educarse juntos, en una misma aula, de modo que puedan interactuar de forma continuada unos y otros" (p.39). Para todos los alumnos la interacción social es fundamental, mientras que el aislamiento de algunos en aulas y programas específicos no hacen más que acrecentar las dificultades de partida, la exclusión dentro del centro y la marginación fuera del mismo. Crear las condiciones para que las interacciones sean satisfactorias debería ser una prioridad en el quehacer cotidiano del profesorado (Avramidis, 2010).

Queda mucho trabajo por delante. Las medidas extraordinarias no dejan de ser "paliativas" dirán Sandoval, Simón y Echeita (2012, p.121), y cuantos más esfuerzos y recursos se ponen en estas, son cada vez menos urgentes las medidas preventivas. Por consiguiente, la desigualdad puede aumentar en los próximos años instaurándose nuevos escenarios de segregación, basados en denostados enfoques y modelos sobre la enseñanza y el aprendizaje.

Son todavía numerosas las sombras que apesadumbran la inclusión educativa, y son pocas las luces si enfocamos a las cuestiones de justicia y derechos sobre las que pivota la educación inclusiva -acentúa Slee (2012). Estas cuestiones también tienen que ver con las barreras a las que aún muchos alumnos han de hacer frente; la falta de equidad o la búsqueda de la misma es la brújula que nos guía a salvaguardar tan preciado valor haciendo visibles las evidencias de la exclusión.

\section{Referencias}

Álvarez, E., Álvarez, M., Castro, P., Campo, M.Á. y Fueyo, E. (2008). Funcionamiento de la integración en la Enseñanza Secundaria Obligatoria según la percepción del profesorado. Psicothema 20(1), 56-61. Recuperado de http://www. psicothema.com/pdf/3429.pdf

Aramendi, P., Vega, A. y Santiago, K. (2011). Los programas de atención a la diversidad en la Educación Secundaria desde la perspectiva de los estudiantes: estudio comparado. Revista de Educación, 356, 185-209. Recuperado de

http://www.revistaeducacion.educacion.es/re356/re356_08.pdf

Avramidis, E. (2010). Social relationships of pupils with special educational needs in the mainstream primary class: peer group membership and peer-assessed social behavior. European Journal o Special Needs Education, 25(5), 413-429. DOI: $10.1080 / 08856257.2010 .513550$

Birbili, M. (2005). Constants and Contexts in Pupil Experience of Schooling in England, France and Denmark. European Educational Research Journal, 4(3), 313-320. Recuperado de

http://journals.sagepub.com/doi/pdf/10.2304/eerj.2005.4.3.10

Danforth, S. y Smith, T.J. (2005). Enganging Troubling Students. A Constructivist Approach. California: Corwin Press.

Domínguez Alonso, J., López Castedo, A. y Vázquez Varela, E. (2016). Atención a la diversidad en la educación secundaria obligatoira: Análisis desde la inspección educativa. Aula Abierta, 44, 70-76. DOI: 10.1016/j.aula.2016.03.002

Durán, D. y Giné, C. (2011). La formación del profesorado para la educación inclusiva: Un proceso de desarrollo profesional y de mejora de los centros para atender la diversidad. Revista Latinoamericana de Inclusión Educativa, 5(2), 153-170. Recuperado de http://www.rinace.net/rlei/numeros/vol5num2/art8.pdf

Echeita, G, Muñoz, Y., Sandoval, M. y Simón, C. (2014). Reflexionando en voz alta sobre el sentido y algunos saberes proporcionados por la investigación en el ámbito de la educación inclusiva. Revista Latinoamericana de Inclusión Educativa, 8(2), 25-48. Recuperado de http://www.rinace.net/ rlei/numeros/vol8-num2/art1.pdf

Escudero, J.M. (2012). La educación inclusiva, una cuestión de derecho. Educatio Siglo XXI, 30(2), 109-128. Recuperado de http://revistas.um.es/educatio/article/view/153711/140751

Escudero, J.M. y Martínez, B. (2012). Las políticas de lucha contra el fracaso escolar: ¿programas especiales o cambios profundos del sistema y la educación? Profesorado. Revista de Educación, núm. extraordinario "Políticas públicas de apoyo y refuerzo educativo", 174-193. DOI: 10.4438/1988-592X-RE2012-EXT-211

Escudero, J.M.; González, M.T. y Martínez, B. (2009). El fracaso escolar como exclusión educativa: comprensión, políticas y prácticas. Revista Iberoamericana de Educación, 50, 41-64. Recuperado de http://rieoei.org/rie50.htm

Fernández Enguita, M., Mena Martínez, L. y Riviére Gómez, J. (2010). Fracaso y abandono escolar en España. Barcelona: Obra Social La Caixa, Colección Estudios Sociales, núm. 29.

Florian, L., Young, K. \& Rouse, M. (2010). Preparing teachers for inclusive and diverse educational environments: studying curricular reform in an initial teacher education course. International Journal of Inclusive Education, 14(7), 709-722. DOI: 10.1080/13603111003778536

Fulcher, G. (1989). Disabling Policies? A Comparative Approach to Education Policy and Disability. London: Falmer.

García Gracia, M., Casal Bataller, J., Merino Pareja, R. y Sánchez Gelabert, A. (2013). Itinerarios de abandono escolar y transiciones tras la Educación Secundaria Obligatoria. Revista de Educación, 361, 65-94. DOI: 10.4438/1988-592X-RE-2011361-135

Garner, P. (2005). Behaviour for Learning: A positive Approach to Managing Classroom Behaviour". In S. Capel, M. Leask y T. Turner (eds.), Learning to Teach in Secondary Schol: A companion to school experience (pp. 136-150). London: Routledge.

Garner, P. y Davies, J.D. (eds.) (2007). Key Questions in Behaviour. Exeter: Leaning Matters.

González, M.T. (2005). El absentismo y el abandono: una forma de exclusion escolar. Profesorado. Revista de Currículum y Formación del Profesorado, 9(1), 1-12. Recuperado de http:// www.ugr.es/ recfpro/rev91ART4res.pdf

Hernández, F. y Tort, A. (2009). Cambiar la mirada sobre el fracaso escolar desde la relación de los jóvenes con el saber. Revista Iberoamericana de Educación, 49(8), 1-11. Recuperado de http://rieoei.org/3109.htm

Leeuw, R.R., de Boer, A.A., Bijstra, J. \& Minnaert, A.E.M.G. (2017). Teacher strategies to support the social participation of students with SEBD in the regular classroom. European Journal of Special Education, DOI: 10.1080/08856257.2017.1334433

López Melero, M. (2004). Construyendo una escuela sin exclusiones. Una forma de trabajar en el aula con proyectos de investigación. Málaga: Aljibe

Marchesi, Á. (2004). Qué será de nosotros los malos alumnos. Madrid: Alianza Editorial.

Mena Martínez, Fernández Enguita, M. y Riviére Gómez, J. (2010). Desenganchados de la educación: procesos, experiencias, motivaciones y estrategias del abandono y del fracaso escolar. Revista de Educación, núm. extraordinario, 119-145. Recuperado de http://www.revistaeducacion.mec. es/re2010/re2010_05.pdf

Meyers, S.A. (2009). Do Your Students Care Whether You Care about them? College Teaching, 57(4), 205-210.

Moliner, O., Sales, A., Ferrández, R., Moliner, L. y Roig, R. (2012). Las medidas específicas de atención a la diversidad 
en la Educación Secundaria Obligatoria (ESO) desde las percepciones de los agentes implicados. Revista de Educación, 358, 197-217. DOI: 10-4438/1988-592X-RE-2010-358-075

Pujolàs, P. (2010). No es inclusión todo lo que se dice que lo es. Aula de Innovación Educativa, 191, 38-41. Recuperado de http:// www.fapac.cat/sites/all/files/aula_1.pdf

Roca, E. (2010). El abandono temprano de la educación y la formación en España. Revista de Educación, núm. extraordinario "Abandono temprano de la educación y la formación: cifras y políticas", 31-62. Recuperado de http:// www.revistaeducacion.mec.es/re2010/re2010_02.pdf

Rodríguez, C., Álvarez, J. y Moreno, Mạ.Á. (2009). Los programas extraordinarios de atención a la diversidad: implicaciones psicológicas, académico-cognitivas y sociales en el alumnado de la comunidad autónoma de Andalucía. Profesorado. Revista de Currículum y Formación del Profesorado, 13(3), 175-192. Recuperado de http://www.ugr.es/ recfpro/rev133ART7.pdf

Rué, J. (coord.) (2006). Disfrutar o sufrir la escolaridad obligatoria. ¿Quién es quién ante las oportunidades escolares? Barcelona: Octaedro.

Sandoval, M. y Simón, C. (2007). Los alumnos con problemas de conducta. Claves para la formación del profesorado. Contextos Educativos, 10, 91-100. Recuperado de https://publicaciones.unirioja.es/ojs/index.php/contextos/article/ view/581

Sandoval, M., Simón, C. y Echeita, G. (2012). Análisis y valoración crítica de las funciones del profesorado de apoyo desde la educación Inclusiva. Revista de Educación, núm. extraordinario "Políticas públicas de apoyo y refuerzo educativo", 117-137. DOI: 10.4438/1988-592X-RE-2012-EXT-209

Saumell, C., Alsina, G. y Arroyo, A. (2011). Alumnado con dificultades de regulación del comportamiento. Vol. I Primaria. Barcelona: Graó.

Slee, R. (2012). La escuela extraordinaria. Exclusión, escolarización y educación inclusiva. Madrid: Morata.

Turner, C. (2006). A pupil with emotional and behavioural difficulties perspective: Does John feel that his behaviour is affecting his learning? Journal Emotional and Behavioural Difficulties, 5(4), 13-18. DOI: 10.1080/1363275000050403

Vilaró, R. (2007). Una Mirada: un retrat de l'educació secundària. Barcelona: Edicions de 1984.

Young, I.M. (2000). Inclusion and Democracy. Oxford: Oxford University Press. 